\title{
O Olhar da População Idosa sobre o Acesso a uma Unidade Básica de Saúde em Porto Alegre - RS
}

The Elderly's Look Regarding the Access to a Basic Health Unit in Porto Alegre - RS

William de Nazareth Nogueira de Oliveira ${ }^{1}$, Lidiane Pivetta Teichmann ${ }^{2}$, Daniel Demétrio Faustino da Silva ${ }^{3}$, Sérgio Antônio Sirena ${ }^{4}$, Margaret Ivanir Schneider ${ }^{5}$

\begin{abstract}
Objective: The aim of this study was to discribe how to give the elderly access to health services in a Basic Health Unit in Porto Alegre since it substantiated data was never available to evaluate the care for the elderly population in this health unit.

Materials and methods: This study was conducted with a descriptive and cross-sectional design, applying semi-structured interviews with fifteen open and closed questions based on PCA-Tool as an instrument in a sample of 61 elderly patients.

Results: The main results show that the Health Unit SESC was remembered by the vast majority of the elderly population as a reference service for basic needs in health. The greatest difficulties of access were related to the characteristics of an area where the Unit is located. It is necessary to consider what measures should be taken to solve this problem. The access of older methods of medical, dental and nursing was considered easier. However, the perception of users about the strategies for prioritization in attendance did not appear so clear.
\end{abstract}

Conclusion: It was concluded from the review of this population that, despite some difficulties pointed out, access to health services was considered easier for the elderly in this Health Unit.

Keywords: health of the elderly; primary health care; health services accessibility

\section{Resumo}

Objetivo: O objetivo deste estudo é descrever como se dá o acesso da população idosa ao serviço de saúde em uma Unidade Básica de Saúde de Porto Alegre, pois nunca se obteve dados fundamentados para avaliar a assistência aos idosos nessa unidade de saúde.

Materiais e métodos: Este estudo foi elaborado de uma forma descritiva e transversal, através de entrevistas semi-estruturadas contendo quinze questões abertas e fechadas baseadas no PCA-Tool como instrumento, com uma amostra de 61 pacientes idosos.

Resultados: A Unidade de Saúde SESC foi lembrada pela grande maioria da população idosa como serviço de referência para primeiras necessidades em saúde. As maiores dificuldades de acesso se deram em relação às características geográficas do território onde a Unidade está inserida. É necessária uma reflexão sobre que medidas devem ser tomadas para solucionar este problema. $\mathrm{O}$ acesso dos idosos às modalidades de consulta médica, odontológica e de enfermagem foi considerado facilitado. Porém, a percepção desses usuários quanto às estratégias de priorização no atendimento não se apresentou tão evidente.

Conclusão: A partir da opinião dessa população, apesar de algumas dificuldades assinaladas, o acesso ao serviço de saúde foi considerado facilitado para os idosos nessa Unidade de Saúde.

Palavras-chave: saúde do idoso; atenção primária a saúde; acesso aos serviços de saúde

Rev. Fac. Odontol. Porto Alegre, v. 51, n. 3, p. 17-22, set./dez., 2010.
${ }^{1}$ Odontólogo Residente, Residência Integrada em Saúde da Família e Comunidade do Grupo Hospitalar Conceição

${ }^{2}$ Enfermeira Residente, Residência Integrada em Saúde da Família e Comunidade do Grupo Hospitalar Conceição

${ }^{3}$ Odontólogo, Serviço de Saúde Comunitária do Grupo Hospitalar Conceição

${ }^{4}$ Médico, Serviço de Saúde Comunitária e Gerência de Ensino e Pesquisa do Grupo Hospitalar Conceição

${ }^{5}$ Enfermeira, Serviço de Saúde Comunitária do Grupo Hospitalar Conceição

Correspondência: William de Nazareth Nogueira de Oliveira

Endereço: Praça Carlos Simão Arnt, 105/1102 - CEP 90450-110, Porto Alegre RS, Brasil

E-mail: dr.williamnogueira@gmail.com

Data de Submissão: 20/11/2011

Data de Aceite: 13/08/2012

\section{Introdução}

A atenção primária à saúde é entendida como uma forma de organização dos serviços de saúde e uma estratégia para integrar todos os aspectos desses serviços, a partir de uma perspectiva da população. Essa perspectiva está em consonância com os princípios do Sistema Único de Saúde (SUS) e tem como diretrizes, no Brasil, a busca por um sistema de saúde voltado a enfatizar a equidade social, a co-responsabilidade entre população e setor público, a solidariedade e um conceito amplo de saúde. Em sua forma mais desenvolvida, a atenção primária deve ser a porta de entrada do sistema de saúde e o local responsável pela organização do cuidado à saúde do paciente e da população ao longo do tempo (DUNCAN et al., 2004).

A prática da atenção primária é, portanto, o exercício de estar à porta de entrada de um sistema de saúde, de ser o primeiro contato de indivíduos, famílias e comunidades com uma complexa rede de serviços, dispensando cuidados às enfermidades, prevenindo os agravos, promovendo a saúde e estimulando o autocuidado, próximo ao cenário onde a população vive o cotidiano de uma sociedade dinâmica e cheia de conflitos (DUNCAN et al., 2004).

Seguindo esse preceito, Starfield (2002), estabelece que a atenção primária à saúde deve ser organizada de forma a obedecer quatro princípios fundamentais: acesso de primeiro contato, longitunalidade, integralidade e coordenação do cuidado.

Paralelamente aos desafios de se proporcionar uma atenção primária de qualidade a todos os usuários do SUS, observamos uma tendência de envelhecimento da população brasileira, o que reforça a necessidade de maior preparo e planejamento dos serviços de saúde para atender as pessoas dessa faixa etária, que possuem características peculiares.

É função das políticas públicas de saúde contribuir para que mais pessoas alcancem as idades avançadas com o melhor estado de saúde possível. O envelhecimento ativo e saudável é o grande objetivo nesse processo. Se considerarmos saúde de forma 
ampliada, torna-se necessária alguma mudança no contexto atual em direção a medidas de prevenção e assistência mais favorável e específica para a população idosa (BRASIL, 2006).

O processo de envelhecimento, que nos países desenvolvidos ocorre de forma gradual, acompanhado de melhorias na cobertura do sistema de saúde, nas condições de habitação, saneamento básico, trabalho e alimentação, no Brasil ocorre num contexto de desigualdades sociais, economia frágil, crescentes níveis de pobreza, com precário acesso aos serviços de saúde e reduzidos recursos financeiros, sem as modificações estruturais que respondam às demandas do novo grupo etário emergente (VERAS, 2002).

A legislação brasileira de proteção à saúde do idoso teve início em dezembro de 1999 quando foi aprovada a Política Nacional de Saúde do Idoso pelo Ministério da Saúde (BRASIL, 1999). Mais tarde, em outubro de 2003, foi criado o Estatuto do Idoso por meio da Lei $n^{\circ}$. 10.741 que assegurou atenção integral à saúde do idoso garantindo atenção especial às doenças que os afetam preferencialmente, incluindo atendimento geriátrico na atenção básica à saúde (BRASIL, 2003; BRASIL, 2006).

Visto esta necessidade de acompanhar este grupo populacional e reduzir os custos de saúde, foi constituída como uma das seis áreas de atuação prioritária nas três esferas de governo, a saúde da população idosa. Isto foi estabelecido dentro do pacto pela vida, locado na portaria $n^{\circ} 399 / 2006$, visando à identificação dos determinantes da saúde do idoso e estabelecendo estratégias que atendam as suas necessidades (BRASIL, 1999; BRASIL, 2003; BRASIL, 2006).

Além disso, os trabalhadores dos serviços de saúde devem estar capacitados em termos de conhecimentos, habilidades e atitudes para elaborar e operar protocolos para ações programáticas específicas às necessidades dos idosos de maneira integrada com as demais práticas da rede de cuidado social (BRASIL, 2006).

A Unidade Básica de Saúde SESC, localizada na zona leste de Porto Alegre-RS e componente do Serviço de Saúde Comunitária do Grupo Hospitalar Conceição (SSC-GHC), tem em seu território de abrangência usuários que apresentam grande desigualdade social entre si. Junto às grandes avenidas, percebemos uma população de classe média e, nas ruas secundárias e becos, uma população beirando a miséria e em convívio constante com a violência ocasionada pelo tráfico de drogas, um cenário típico das grandes cidades brasileiras. Apesar de não ser uma Unidade de Saúde pertencente à Estratégia de Saúde da Família, o processo de trabalho elaborado por ela e por todas as outras onze Unidades do SSC-GHC seguem a lógica de uma atenção focada na prevenção e promoção da saúde, considerando as características da população residente em um território definido e sempre buscando ao máximo aprimorar o atendimento de forma a obedecer todos os princípios da atenção primária à saúde.

A Unidade de Saúde demonstrou não possuir dados documentados sobre a assistência oferecida à população idosa do seu território de abrangência. Além disso, há o desconhecimento sobre as peculiaridades dessa população e se essa assistência tem seguido adequadamente os princípios norteadores da Atenção Primária à Saúde, segundo Starfield (2002) (acesso de primeiro contato; longitudinalidade; integralidade e coordenação do cuidado). Assim, a avaliação da população idosa sobre a atenção à saúde prestada a ela, é imprescindível para que se consiga melhorar a qualidade do atendimento e praticar medidas preventivas. Por isso, o objetivo deste estudo foi descrever como se dá o acesso da população idosa ao serviço de saúde em uma Unidade Básica de Saúde de Porto Alegre.

\section{Materiais e Métodos}

Trata-se de um estudo descritivo transversal e de análise quantitativa dos dados, utilizando uma amostra da população idosa, com idade acima de 60 anos e cadastrada na Unidade de Saúde SESC.

O universo populacional, dessa faixa etária, registrado no Sistema de Informações da Unidade de Saúde foi de 660 pessoas e utilizando o Software Epi-Info para cálculo amostral (com nível de confiança de $99 \%$, frequência esperada de $20 \%$ e pior resultado aceitável de $10 \%$ ), ficou estipulada uma amostra de 61 usuários a serem entrevistados.

Foram incluídos os usuários idosos em condições cognitivas suficientes para responder ao questionário e que estavam cadastrados na unidade há mais de um ano. Os idosos acamados e atendidos pelo Programa de Atenção Domiciliar- PAD foram excluídos da amostra, por terem uma atenção diferenciada.

A amostra foi sorteada em número proporcional a cada uma das cinco áreas de vigilância do território correspondente a unidade, que está sob responsabilidade de um Agente Comunitário de SaúdeACS. Antes foi feita a adequação da lista, aplicando os critérios de inclusão e exclusão. Cada usuário sorteado era investigado anteriormente junto à ACS de referência para saber se essa pessoa tem consultado regularmente na Unidade de Saúde. Em algumas situações foi necessária a busca dessas informações em prontuário de família.

No decorrer da busca pelos usuários idosos sorteados para participar da amostra foram acontecendo problemas, desde a recusa à participação na pesquisa ( 3 pessoas) até a dificuldade de encontrar essas pessoas em seus domicílios (8 pessoas), devido aos mais diversos fatores. Nesses casos, o próximo usuário da lista que residia na mesma microárea do território era selecionado.

$O$ instrumento consistiu em uma entrevista semi-estruturada com trinta e cinco questões abertas e fechadas, baseadas no PCATool, uma ferramenta de avaliação dos princípios da atenção primária à saúde (HARZHEIM et al., 2006; HARZHEIM, DUNCAN, OLIVEIRA, 2007), contemplando questionamentos acerca do atendimento prestado pela equipe da Unidade de Saúde SESC em relação aos princípios básicos da APS (acesso, longitudinalidade, integralidade e coordenação do cuidado). Algumas questões abordaram especificamente $o$ atendimento realizado pela equipe de enfermagem, médicos e odontólogos.

O presente artigo é parte integrante de uma pesquisa maior que analisou todos os atributos da atenção primária à saúde em relação à população idosa. Neste, serão discutidos apenas os dados referentes às quinze questões sobre o acesso ao serviço de saúde, devido à grande quantidade de informações coletadas.

Foi realizado um estudo piloto com dez usuários idosos, que foram se consultar na Unidade de Saúde por qualquer motivo, com o intuito de aperfeiçoar o instrumento da entrevista a ser realizada.

As entrevistas foram realizadas por dois pesquisadores durante os meses de maio a agosto de 2010, em visitas domiciliares, em local reservado dentro do domicílio, que garantia sigilo e privacidade aos usuários. O tempo dispensado com cada entrevista foi de aproximadamente trinta minutos.

O projeto de pesquisa foi aprovado pelo Comitê de Ética em Pesquisa do Grupo Hospitalar Conceição, e seguindo as determinações éticas presentes na Resolução 196/1996, em que todos os sujeitos envolvidos assinaram o Termo de Consentimento Livre e Esclarecido -TCLE.

Os dados obtidos foram codificados, digitados e analisados com auxílio do pacote estatístico EPI INFO versão 3.5.1/2008 e serão

Rev. Fac. Odontol. Porto Alegre, v. 51, n. 3, p. 17-22, set./dez., 2010. 
apresentados na forma de estatística descritiva (freqüência absoluta e relativa) através de tabelas.

\section{Resultados e Discussão}

Conforme dados da Tabela 1, dos 61 usuários idosos entrevistados, em relação a qual serviço de saúde procuram primeiro, $51(83,6 \%)$ relataram ir inicialmente à Unidade de Saúde SESC sempre que adoecem. 3 pessoas $(4,9 \%)$, procuram diretamente o Hospital Nossa Senhora da Conceição para serem atendidos, mesmo no horário em que a unidade de saúde se encontra aberta, pois entendem que o acesso e a resolutividade de seu problema são mais facilitados no atendimento hospitalar. O mesmo número de idosos, 3 $(4,9 \%)$, procuram atendimento diretamente em clínica particular, em função de uma possível dificuldade no acesso ao serviço oferecido pela Unidade de Saúde e pelo SUS.

Existem ainda aqueles que procuram outros serviços de emergência como o Hospital Ernesto Dorneles (1 pessoa - 1,6\%), Hospital da PUCRS (1 pessoa - 1,6\%) e Unidade de Saúde Bom Jesus (1 pessoa - 1,6\%), que apesar de ser um posto de saúde, oferece atendimento de urgência 24 horas, na lógica de pronto atendimento semelhante a uma unidade hospitalar. A Unidade de Saúde Vila Ipiranga foi citada por uma pessoa $(1,6 \%)$, sendo relevante $o$ fato de esse serviço estar situado tangencialmente ao limite do território definido da Unidade SESC, sendo assim, algumas pessoas residem muito mais próximas dessa unidade em relação à Unidade SESC. Outros usuários que moram próximo da Unidade de Saúde Vila Ipiranga também foram entrevistados e mesmo com a facilidade de acesso territorial devido à proximidade de suas casas, ainda preferem se deslocar até a Unidade de Saúde SESC por entender que serão mais bem atendidos.

Tabela 1 - Distribuição dos serviços de saúde referidos pelos idosos como local do primeiro atendimento.

\begin{tabular}{lcc}
\hline & $\mathrm{n}$ & $\%$ \\
\hline Unidade SESC & 51 & 83,6 \\
Clínica ou consultório particular & 3 & 4,9 \\
Hospital Nossa Senhora da & 3 & 4,9 \\
Conceição & 1 & 1,6 \\
Unidade Vila Ipiranga & 1 & 1,6 \\
Hospital São Lucas da PUC-RS & 1 & 1,6 \\
Hospital Ernesto Dorneles & 1 & 1,6 \\
Unidade Bom Jesus & & \\
\hline
\end{tabular}

A Tabela 2 demonstra os dados em relação à facilidade de acesso aos diversos tipos de consulta médica, odontológica e de enfermagem. No que se refere ao atendimento de urgência para consulta médica (consulta solicitada no dia, sem agendamento prévio) 42 pessoas $(68,9 \%)$ relataram achar fácil o acesso, enquanto 11 pessoas (18\%) consideraram difícil e 8 pessoas $(13,1 \%)$ afirmaram nunca ter necessitado desse tipo de atendimento com a medicina. Ao considerar apenas os 53 usuários que necessitaram desse tipo de consulta, $79,2 \%$ (42 pessoas) afirmaram conseguir esse tipo de atendimento com facilidade.
Tabela 2 - Distribuição dos usuários idosos quanto à facilidade de acesso às consultas médicas, odontológicas e de enfermagem.

\begin{tabular}{|c|c|c|c|}
\hline & Fácil & Difícil & $\begin{array}{c}\text { Nunca } \\
\text { precisou }\end{array}$ \\
\hline Tipos de acesso & $\mathrm{n}(\%)$ & $\mathrm{n}(\%)$ & $\mathrm{n}(\%)$ \\
\hline $\begin{array}{l}\text { Acesso à consulta } \\
\text { médica de urgência }\end{array}$ & $42(68,9)$ & $11(18)$ & $8(13,1)$ \\
\hline $\begin{array}{l}\text { Acesso à consulta } \\
\text { odontológica de } \\
\text { urgência }\end{array}$ & $22(36,1)$ & $3(4,9)$ & $36(59)$ \\
\hline $\begin{array}{l}\text { Acesso à } \\
\text { enfermagem }\end{array}$ & $35(57,4)$ & $0(0)$ & $26(42,6)$ \\
\hline $\begin{array}{l}\text { Acesso à consulta } \\
\text { médica programada }\end{array}$ & $54(88,5)$ & $5(8,2)$ & $2(3,3)$ \\
\hline $\begin{array}{l}\text { Acesso à consulta } \\
\text { odontológica } \\
\text { programada }\end{array}$ & $30(49,2)$ & $2(3,3)$ & $29(47,5)$ \\
\hline $\begin{array}{l}\text { Acesso à consulta } \\
\text { de enfermagem } \\
\text { programada }\end{array}$ & $14(23)$ & $1(1,3)$ & $46(75,4)$ \\
\hline $\begin{array}{l}\text { Acesso à consulta } \\
\text { de retorno com o } \\
\text { médico }\end{array}$ & $56(91,8)$ & $2(3,3)$ & $3(4,9)$ \\
\hline $\begin{array}{l}\text { Acesso à consulta } \\
\text { odontológica } \\
\text { programada de } \\
\text { retorno }\end{array}$ & $33(54,1)$ & $1(1,6)$ & $27(44,3)$ \\
\hline $\begin{array}{l}\text { Acesso à consulta } \\
\text { de enfermagem } \\
\text { programada de } \\
\text { retorno }\end{array}$ & $12(19,7)$ & $0(0)$ & $49(80,3)$ \\
\hline
\end{tabular}

Considerando as urgências odontológicas (Tabela 2), 22 pessoas $(36,1 \%)$ afirmaram que é fácil conseguir atendimento com o dentista quando estão com dor de dente ou possuem alguma necessidade que não possa esperar. Apenas 3 pessoas $(4,9 \%)$ consideraram difícil o acesso a esse serviço e 36 pessoas (59\%) relataram nunca ter precisado de atendimento odontológico de urgência.

Se levarmos em consideração apenas os 25 usuários que sentiram a necessidade de ir ao dentista em consulta de urgência, $88 \%$ (22 pessoas) relataram facilidade em acessar o serviço dessa forma.

Nota-se que a maioria dos usuários entrevistados relatou não precisar de consulta odontológica de urgência. Isso pode ser explicado pela falta de percepção da necessidade odontológica sentida pelos idosos ou pela realidade da condição bucal desdentada parcialmente ou totalmente, típica da população idosa brasileira. Ou por outro lado, a baixa procura dessa modalidade de consulta também pode ser explicada pelo fato da população idosa ser mais disciplinada e com maior disponibilidade de tempo, buscando com mais frequência o serviço odontológico em consultas agendadas.

Conseguir atendimento sem agendamento prévio com a equipe de enfermagem foi considerado fácil por 35 pessoas $(57,4 \%)$ e 26 pessoas $(42,6 \%)$ relataram nunca ter precisado desse tipo de serviço (Tabela 2). Dos usuários que precisaram desse tipo de 
serviço nenhum comentou ter sido difícil o acesso à equipe de enfermagem da unidade de saúde. Isso se explica pela grande disponibilidade da equipe de enfermagem, que é responsável pelo acolhimento ao usuário, em escutar e resolver todos os casos demandados pela população idosa.

Sob o ponto de vista das consultas programadas com os médicos, 54 usuários $(88,5 \%)$ afirmaram ter sido fácil conseguir consulta; 5 usuários $(8,2 \%)$ relataram não ter sido fácil e 2 usuários $(3,3 \%)$ disseram nunca ter necessitado de consulta agendada com algum médico (Tabela 2). Os índices ainda melhoram ao considerar só os 59 usuários que disseram precisar desse tipo de atendimento, com $91,5 \%$ de aprovação em relação à facilidade no acesso.

Desde abril de 2009, os pacientes idosos têm privilégio na marcação de consultas, integrando uma fila preferencial, sendo passados ao atendimento anteriormente para conseguir marcar consultas de forma mais facilitada. Além disso, os usuários idosos vinculados aos programas de prevenção às doenças como hipertensão e diabetes, por exemplo, acabam também se beneficiando junto aos outros usuários, possuindo um tipo de agendamento diferenciado, com suas consultas disponibilizadas em horários específicos reservados para marcar com o médico.

As consultas odontológicas programadas (Tabela 2) foram de fácil acesso a 30 usuários $(49,2 \%)$ e difícil para 2 usuários $(3,3 \%)$, sendo ainda que, 29 usuários $(47,5 \%)$ afirmaram nunca ter precisado do atendimento odontológico agendado. Esses dados demonstram que quase metade dos usuários não procura o atendimento seguindo uma lógica preventiva e ainda vinculam a consulta programada com o dentista a uma necessidade sentida por dor ou estética muito comprometida. Muitos usuários entrevistados são portadores de próteses totais e acreditam no fato de não precisar consultar com o dentista por não possuir mais dentes. Pode-se inferir também que a população idosa talvez já esteja coberta quanto as suas maiores necessidades em saúde bucal durante a vida, não percebendo mais a necessidade de procurar atendimento.

Além disso, muitos idosos ainda vinculam o atendimento odontológico às más experiências antigas vivenciadas, evitando buscar a odontologia por lembrar as práticas clínicas executadas antigamente, quando eram submetidos na maioria das vezes a procedimentos de exodontia e restaurações com presença de dor.

Se apenas os 32 usuários entrevistados que relataram precisar de consulta odontológica programada forem considerados, o índice de aprovação ao acesso aumenta consideravelmente, atingindo $93,75 \%$ (30 pessoas).

Quanto às consultas programadas com as enfermeiras (Tabela 2), 46 usuários $(75,4 \%)$ afirmaram nunca ter necessitado desse atendimento. Se considerarmos apenas os 15 usuários que relataram necessitar desse tipo de atendimento, 93,3 \% (14 pessoas) entenderam que foi fácil conseguir a consulta de enfermagem.

A maioria dos idosos que procura o serviço de enfermagem é atendida diretamente pelas enfermeiras e pelos auxiliares de enfermagem sem agendamento prévio, sendo realizados principalmente procedimentos como curativos e aferição da tensão arterial e glicemia. Nas consultas programadas com as enfermeiras geralmente são realizadas, por exemplo, coletas de citopatológico para exame preventivo de câncer de colo do útero, assim como orientações de pré-natal e nutricional para gestantes e mães de crianças recém-nascidas. Portanto, são consultas que na maioria das vezes não atingem a população idosa, o que explica o fato de a maioria dos usuários não demonstrar sentir necessitar dessa modalidade de consulta.

Foram analisadas também as consultas de retorno/manutenção realizadas pelos médicos, dentistas e enfermeiras.

A Tabela 2 demonstra, ainda, que 56 pessoas $(91,8 \%)$ relataram facilidade em conseguir consulta de retorno com o médico,
2 pessoas (3,3\%) consideraram difícil e 3 pessoas (4,9\%) afirmaram nunca ter necessitado acessar novamente o serviço médico através da consulta de retorno.

A facilidade em conseguir as consultas de retorno com o médico ainda aumenta se forem considerados apenas os pacientes que relataram precisar de acompanhamento periódico. Desses 58 usuários, $96,5 \%$ (56 pessoas) alegaram ser fácil o acesso a esse tipo de consulta.

Os pacientes em acompanhamento clínico geralmente têm sua consulta de retorno garantida ao sair do consultório médico. Quando não é marcado já um dia definido na agenda, os usuários idosos da unidade que, na maioria das vezes, tem sua condição de saúde controlada pelo programa de hipertensos e diabéticos, são orientados a retornar à unidade de saúde a partir de um dia alternativo do mês, evitando entrar na fila comum do dia da marcação de consultas gerais.

Dos 61 usuários idosos entrevistados, 27 (44,3\%) relataram nunca ter precisado de consulta de retorno com o dentista e 33 $(54,1 \%)$ consideraram fácil o acesso novamente ao atendimento para manutenção do tratamento. Apenas 1 usuário $(1,6 \%)$ afirmou ter sido difícil acessar o retorno ao atendimento odontológico (Tabela 2).

$\mathrm{O}$ atendimento odontológico tem a peculiaridade de ser um serviço em que praticamente não existe alta para o paciente, pois a probabilidade dele ter que retornar dentro de alguns meses para reavaliação é muito grande. Considerando esse aspecto as consultas de retorno/manutenção são oferecidas aos usuários sempre que finalizam o tratamento durante um determinado momento.

Como já destacado anteriormente, é relevante o número de pacientes que afirmaram não necessitar mais do atendimento odontológico. O alto índice de idosos portadores de próteses totais pode ser novamente o motivo da falta de necessidade sentida em retornar ao dentista. Culturalmente, a população idosa ainda procura o dentista para tratar apenas os dentes e , quando não os possui mais, deixa de frequentar o consultório odontológico. Isso pode ser considerado um fator de risco para o desenvolvimento de patologias bucais, dentre elas o câncer bucal, pois o acompanhamento periódico das lesões ocasionadas ou não pelo uso de próteses dentárias tende à prevenção.

Dessa forma, considerando apenas os 34 usuários que relataram precisar do retorno ao atendimento odontológico, 97\% acreditam ser fácil conseguir acesso a esse tipo de consulta.

A consulta de retorno com a enfermeira não teve a necessidade sentida para 49 pessoas $(80,3 \%)$. Todas as 12 pessoas que afirmaram precisar acessar novamente o atendimento com a enfermeira concordaram ser fácil o retorno às consultas (Tabela 2).

As consultas programadas para idosos com a enfermeira, geralmente não seguem uma lógica de acompanhamento ao longo do tempo, sendo pouco frequentes e, quando ocorrem, o paciente recebe alta ou fica sendo acompanhado pela equipe de auxiliares de enfermagem, que não foi avaliada no questionário.

Portanto, nenhum usuário relatou achar difícil consultar com a sua enfermeira de referência novamente depois de um determinado período.

Os usuários também foram questionados se são atendidos no mesmo dia em que procuram o serviço, quando a unidade de saúde está aberta. 54 usuários $(88,5 \%)$ afirmaram que são atendidos no mesmo dia e 7 usuários $(11,5 \%)$ relataram que não são atendidos (Tabela 3). Isso demonstra a postura acolhedora dos profissionais da Unidade, que tentam ao máximo atender a necessidade de todos. Porém, alguns usuários acabam sendo orientados a retornar a Unidade em outro momento devido à impossibilidade de atendimento. 
Tabela 3 - Distribuição dos usuários idosos quanto ao tempo de espera e priorização no atendimento.

$\begin{array}{cc}\operatorname{Sim} & \text { Não } \\ \mathrm{n}(\%) & \mathrm{n}(\%)\end{array}$

Atendimento no mesmo dia em que procuram a unidade de saúde

$57(88,5) \quad 7(11,5)$

Sentem-se priorizados no atendimento

$36(59)$

$25(41)$

Longo tempo de espera e a necessidade de falar com muitas pessoas para conseguir uma consulta

$7(11,5) \quad 54(88,5)$

Quando questionados se sentem que são priorizados no atendimento por ser idoso, 36 usuários (59\%) afirmaram que sim e 25 usuários (41\%) que não (Tabela 3 ). Muitos idosos comentaram ser normal o fato de não serem priorizados no atendimento, inclusive considerando que se sentiriam constrangidos se houvesse algum tipo de facilidade específica para eles no acesso ao atendimento. Além disso, observou-se que as pessoas que recentemente entraram na faixa dos 60 anos têm dificuldade de se assumir como idosos, pois nossa cultura ainda insiste em caracterizar a figura do idoso através de uma forma fragilizada, depreciativa ou desrespeitosa, o que faz as pessoas se esquivarem dessa associação.

Apesar das medidas de priorização do acesso oferecidas pela Unidade, não se observa claramente a percepção das mesmas pelos usuários, bem como a importância delas para facilitar a entrada no serviço de saúde. Talvez as prioridades garantidas legalmente não tenham chegado ao conhecimento da população idosa ao ponto de sentirem o impacto positivo em relação à sua utilização.

Apenas 7 pessoas $(11,5 \%)$ relataram ter que esperar muito tempo ou falar com muitas pessoas para conseguir uma consulta, enquanto 54 pessoas $(88,5 \%)$ afirmaram não passar por esse tipo de problema quando sentiram necessidade de atendimento na Unidade de Saúde (Tabela 3). Esses dados podem demonstrar indiretamente que o processo gerencial da Unidade está adequado à demanda de pacientes idosos.

A dificuldade no acesso pode ocorrer também em consequência de problemas decorrentes de características do território onde os usuários idosos vivem e onde a Unidade de Saúde está inserida. Louvison et. al (2008) analisaram as desigualdades no uso e acesso aos serviços de saúde entre idosos do município de São Paulo no ano de 2000 e constatou que, quando analisados os idosos de menor renda, as principais dificuldades de acesso citadas foram a distância e qualidade dos serviços de saúde.

Até o ano de 2005 a Unidade de Saúde era localizada em uma casa alugada, em outra rua, mais próxima a uma avenida principal do bairro, um pouco distante das áreas de risco do território. Atualmente está inserida em um terreno próprio, localizado no alto de um morro, próxima às áreas de risco e pontos de tráfico de drogas.

Considerando estes fatos, algumas pessoas idosas evitam ir até a Unidade procurar atendimento, por acharem distante de sua casa e/ou de difícil acessibilidade geográfica ou por sentirem insegurança, devido à presença de traficantes na região.
Nesse contexto, quando perguntados se apresentam alguma dificuldade de ir até a Unidade de Saúde (Tabela 4) a resposta foi positiva para 24 pessoas $(39,3 \%)$ e negativa para 37 pessoas $(60,7 \%)$. Essas limitações foram classificadas em 3 tipos : Distância da sua casa até a Unidade de Saúde; Barreiras geográficas que pudessem interferir no deslocamento até a Unidade de Saúde (lombas, valões etc...); e situações sociais já diagnosticadas no território, como a possibilidade de assaltos e violência causada, principalmente, pela desavença entre grupos rivais pelo controle do tráfico de drogas.

Listadas as alternativas, das 24 pessoas que relataram apresentar alguma dificuldade de ir até a Unidade de Saúde, 9 $(37,5, \%)$ disseram que as barreiras geográficas são a causa maior de limitação no acesso. 9 pessoas $(37,5 \%)$ associaram também as barreiras geográficas à distância grande de sua casa até a Unidade de Saúde. 3 idosos (12,5\%) afirmaram que suas dificuldades são o conjunto das três alternativas (distância, situações sociais e barreiras geográficas); 2 usuários (8,3\%) relataram que encaram a distância como a única dificuldade de acesso e apenas 1 usuário $(4,2 \%)$ adicionou as situações sociais à distância (Tabela 4).

Esses dados nos fazem refletir sobre o papel do poder público em planejar melhor o local de construção das Unidades de Saúde, bem como definir adequadamente o seu território de abrangência para que o serviço de saúde seja disponibilizado em uma região centralizada que facilite o acesso de todos os usuários, não deixando de considerar também os fatores sócio-culturais adquiridos pela população daquele determinado local.

Tabela 4 - Distribuição das dificuldades de deslocamento até a Unidade de Saúde.

\begin{tabular}{|c|c|c|}
\hline & $\mathrm{n}$ & $\%$ \\
\hline Apresenta dificuldade(s) & 24 & 39,3 \\
\hline Não apresenta dificuldade(s) & 37 & 60,7 \\
\hline Total & 61 & 100 \\
\hline Tipos de dificuldade & $\mathrm{n}$ & $\%$ \\
\hline Distância & 2 & 8,3 \\
\hline Barreiras geográficas & 9 & 37,5 \\
\hline Distância e barreiras geográficas & 9 & 37,5 \\
\hline Distância e situações sociais & 1 & 4,2 \\
\hline $\begin{array}{l}\text { Distância, barreiras e situações } \\
\text { sociais }\end{array}$ & 3 & 12,5 \\
\hline Total & 24 & 100 \\
\hline
\end{tabular}

\section{Conclusão}

O estudo demonstrou que a maioria dos usuários acima de 60 anos de idade atendida no serviço está sendo contemplada com uma atenção primária à saúde de qualidade, pelo menos no que se diz respeito ao acesso como porta de entrada para o atendimento pelo Sistema Único de Saúde.

A Unidade de Saúde SESC foi lembrada pela grande maioria da população idosa como serviço de referência para primeiras necessidades em saúde. 
O acesso dos idosos às modalidades de consulta médica, odontológica e de enfermagem foi considerado facilitado. Porém, a percepção desses usuários quanto às estratégias de priorização no atendimento não se apresentou tão evidente. As maiores dificuldades de acesso se deram em relação às características geográficas do território onde a Unidade está inserida.

\section{Referências}

BRASIL. Ministério da Previdência Social. Lei $\mathrm{n}^{\circ}$. 10.741 , de $1^{\circ}$ de outubro de 2003. Dispõe sobre o Estatuto do Idoso e dá outras providências. Disponível em: <www.ufrgs.br/3idade/legis.html>. Acesso em: out. 2009.

BRASIL. Ministério da Saúde. Portaria 1395/GM, de 10 de dezembro de 1999. Aprova a Política Nacional do Idoso. Disponível em: <www.ufrgs.br/3idade/legis.html>. Acesso em: out. 2009.

BRASIL. Ministério da Saúde. Secretaria de Atenção à Saúde. Departamento de Atenção Básica. Envelhecimento e saúde da pessoa idosa. Brasília, 2006. 192p. (Cadernos de atenção básica; $n$. 19).

BRASIL. Ministério da Saúde. Secretaria da Atenção à Saúde. Departamento de Atenção Básica. Política Nacional de Atenção Básica. Brasília, 2006. (Série pactos pela saúde; v. 4).

DUNCAN, B.B. et al. Medicina ambulatorial: condutas de Atenção Primária baseadas em evidências. Porto Alegre: Artmed, 2004. 1600p.

HARZHEIM, E. et al. Consistência interna e confiabilidade da versão em português do instrumento de avaliação da Atenção Primária (PCATool-Brasil) para serviços de saúde infantil. Cad. Saúde Pública, Rio de Janeiro, v. 22, n. 8, p. 1649-1659, ago. 2006.

HARZHEIM, E.; DUNCAN, B.B.; OLIVEIRA, M.M.C.; AGOSTINHO, M. Validação do Instrumento de Avaliação da Qualidade da Atenção Primária- PCATool-Adulto; 2007.

A referência está incompleta. Não foi possível completá-la, pois não a localizei.

LOUVISON, M.C.P. et al. Desigualdades no uso e acesso aos serviços de saúde entre idosos do município de São Paulo. Rev. Saúde Pública, São Paulo, v. 42, n. 4, p. 733-740, ago. 2008.

STARFIELD, B. Atenção Primária: equilíbrio entre necessidade de saúde, serviços e tecnologia. Brasília: UNESCO: Ministério da Saúde, 2002. 726 p.

VERAS, R.P. Terceira idade: gestão contemporânea em saúde. Rio de Janeiro: Relumé-Dumará, 2002. 190 p.

Rev. Fac. Odontol. Porto Alegre, v. 51, n. 3, p. 17-22, set./dez., 2010. 